TITLE:

\title{
One-step fabrication of Au@Pd core-shell bimetallic nanofibers at the interface between water and redox-active ionic liquid
}

\section{$\operatorname{AUTHOR}(\mathrm{S}):$}

Zhang, Yu; Nishi, Naoya; Sakka, Tetsuo

\section{CITATION:}

Zhang, Yu ...[et al]. One-step fabrication of Au@Pd core-shell bimetallic nanofibers at the interface between water and redox-active ionic liquid. Electrochimica Acta 2019, 325: 134919.

ISSUE DATE:

2019-12-01

URL:

http://hdl.handle.net/2433/254666

\section{RIGHT:}

(c) 2019. This manuscript version is made available under the CC-BY-NC-ND 4.0 license

http://creativecommons.org/licenses/by-nc-nd/4.0/:; The full-text file will be made open to the public on 1 December 2021 in accordance with publisher's 'Terms and Conditions for Self-Archiving'.; This is not the published version. Please cite only the published version.; この論文は出版社版でありません。引用の際には出版社版をご確認ご利用ください。 


\section{One-step fabrication of Au@Pd core-shell bimetallic nanofibers at the interface between water and redox-active ionic liquid}

Yu Zhang, ${ }^{[a]}$ Naoya Nishi ${ }^{*[a]}$ and Tetsuo Sakka ${ }^{[a]}$

[a] Y. Zhang, N. Nishi, T. Sakka

Department of Energy and Hydrocarbon Chemistry, Graduate School of Engineering, Kyoto University.

Kyoto 615-8510, Japan.

E-mail: nishi.naoya.7e@kyoto-u.ac.jp 


\section{Abstract}

Au@Pd core-shell bimetallic nanofibers (BNFs) have been successfully prepared with a one-step fabrication method, where no additional preparation step using a template is required. The preparation is realized by the spontaneous growth of Au@Pd core-shell bimetallic nanofibers at the interface between water (W) and a hydrophobic redox-active ionic liquid (RAIL). The RAIL plays dual roles of reducing agent to reduce metal precursors dissolved in $\mathrm{W}, \mathrm{AuCl}_{4}{ }^{-}$and $\mathrm{PdCl}_{4}{ }^{2-}$, at the $\mathrm{RAIL} \mid \mathrm{W}$ interface, as well as the hydrophobic liquid phase constituting the RAIL-W two-phase system. The reduction reactions of $\mathrm{AuCl}_{4}{ }^{-}$and $\mathrm{PdCl}_{4}{ }^{2-}$ at the RAIL/W interface proceed sequentially; $\mathrm{AuCl}_{4}{ }^{-}$is reduced prior to $\mathrm{PdCl}_{4}{ }^{2-}$ to produce $\mathrm{Au}$ nanofibers, which act as the core for the following deposition and growth of Pd shell on the Au surface. Thus, the reductive formation of Au as the core and that of Pd as the shell can be automatically completed in one step. The mechanisms for the interfacial charge transfer reactions and the growth of Au@Pd BNFs are discussed in detail. Control experiments have clearly confirmed that the RAIL greatly promotes the reduction of $\mathrm{PdCl}_{4}{ }^{2-}$ and prevents the agglomeration of Au@Pd BNFs. The prepared Au@Pd BNFs exhibit higher electrocatalytic performance towards ethanol oxidation reaction than the commercial $\mathrm{Pd} / \mathrm{C}$ catalyst, and the catalytic ability is even improved after long-term cycles. TEM images reveal a structural transformation of the Pd shell in the Au@Pd BNFs after long-time cycles, which is responsible for the increased catalytic ability.

Keywords: ionic liquid|water interface; electron transfer; ion transfer; ITIES; electrodeposition. 


\section{Introduction}

Noble metal nanostructures show excellent catalytic performance in a variety of catalytic reactions and therefore, are of great significance in the field of catalysis. In the past decades, noble metal nanostructures with various morphology have been prepared $^{[1-4]}$. One dimensional (1D) nanostructures, including nanowires ${ }^{[5-7]}$, nanofibers ${ }^{[8-10]}$, and nanotubes ${ }^{[11-13]}$, have drawn great attention of researchers by virtue of their unique properties and outstanding application performance. It was reported that the networks formed by 1D nanostructures inherently possess electronic conduction pathways and hence exhibit superior application potential compared with 0D nanoparticles ${ }^{[14]}$, Bimetallic nanofibers (BNFs) incorporate the particular advantages of $1 \mathrm{D}$ structures with their distinctive properties of bimetal, which are remarkably different from those of monometallic counterparts, and thus have attracted extensive interest ${ }^{[15-17]}$. Zhu et al. have prepared PdM (M = Au, Pt) bimetallic alloy nanowires by using Te nanowires as a sacrificial template and reducing agent ${ }^{[18]}$. Hong et al. synthesized dendritic $\mathrm{Au} / \mathrm{Pt}$ and $\mathrm{Au} / \mathrm{PtCu}$ nanowires by means of epitaxial growth of Pt and PtCu dendritic nanoshell on Au nanowires, which were prepared by using Te nanowires as the template ${ }^{[19]}$. To date, frequently-used synthesis strategies rely on such templates ${ }^{[20-23]}$, and therefore, inevitably encounter the preparation of templates as the first step, which increases the difficulties and also time and energy spent during the synthesis process. By far, there still lacks a facile and one-step method to directly prepare BNFs.

Apart from the conventional one-phase solution strategies, the oil|water $(\mathrm{O} \mid \mathrm{W})$ interfacial method is also widely adopted for the preparation of noble metal nanostructures ${ }^{[24-27]}$. It is carried out by separating the reducing agent only soluble in one phase and metal precursor only soluble in the other phase, spatial-selectively 
confining the redox reaction that takes place only at the interface. The organic solvents in the $\mathrm{O} \mid \mathrm{W}$ interfacial method can be replaced to ionic liquids (ILs), which are liquid salts entirely composed of cations and anions ${ }^{[28-30]}$. In previous reports, we have successfully prepared noble metal nanofibers by applying the IL/W interfacial method ${ }^{\text {[31-34] }}$. Moreover, due to the great difference of the properties between $\mathrm{W}$ and IL, the IL $\mid W$ interface provides formation sites for metal to grow towards each liquid phase with different morphology, and makes it possible to connect them together ${ }^{[31-34]}$. Besides, by elaborate designs of the chemical structures of IL-ions, ILs can be endowed with multiple functions in the IL/W system rather than just as a solvent. For instance, IL-ions form rigid multilayering structures at the interface ${ }^{[35-39]}$ and prevent the agglomeration of metal nanostructures. When an IL contains redox-active group in the constituting ion, the IL itself can be the reducing agent for metal precursor and liquid phase in the IL/W system simultaneously. Samec et al. employed a redox active IL (RAIL), (ferrocenylmethyl)dodecyldimethylammonium tetrakis[3,5bis(trifluoromethyl)phenyl]borate ([FCMDDA $\left.{ }^{+}\right]\left[\mathrm{TFPB}^{-}\right]$), to form the electrochemically polarizable RAIL/W interface, and measured the electron transfer (ET) across the RAIL|W interface between FcMDDA ${ }^{+}$constituting RAIL and redox species dissolved in $\mathrm{W}^{[40,41]}$. Our previous study has adopted the interface between the RAIL (ferrocenylmethyl)dodecyldimethylammonium bis(nonafluorobutanesulfonyl)amide ([FcMDDA $\left.\left.{ }^{+}\right]\left[\mathrm{C}_{4} \mathrm{C}_{4} \mathrm{~N}^{-}\right]\right)$and water as the reaction site to prepare vertically aligned Pd nanofiber arrays without the use of template ${ }^{[34]}$. Other than as the reducing agent, the RAIL has been confirmed to play an indispensable role for the spontaneous formation of Pd nanofiber arrays ${ }^{[34]}$.

In this study, we present a one-step fabrication strategy to prepare Au@Pd coreshell BNFs, where an additional preparation step of the template is not required. The hydrophobic RAIL, $\left[\mathrm{FCMDDA}^{+}\right]\left[\mathrm{TFPB}^{-}\right]^{[40,41]}$ was employed as the reducing agent 
for metal precursors as well as the hydrophobic liquid phase to form the electrochemically polarizable RAIL|W interface, which was served as the formation sites for the spontaneous growth of Au@Pd BNFs. The reaction-time dependence of the morphology revealed that Au nanofibers were formed preferentially and acted as the core for the following growth of Pd shell on the Au surface. Control experiments confirmed that the RAIL not only plays a role of the reducing agent, but also greatly promote the reduction of Pd and prevent the agglomeration of Au@Pd NBFs. The prepared Au@Pd BNFs show excellent electrocatalytic performance toward ethanol oxidation reaction (EOR) which surpassed the commercial $\mathrm{Pd} / \mathrm{C}$ catalyst, and an improved catalytic ability after long-term cycles. The prepared Au@Pd BNFs have good application potential for the field of the direct ethanol fuel cell.

\section{Experimental}

\subsection{Synthesis of Au@Pd BNFs}

For a typical synthesis, $2 \mathrm{~mL}$ of $\mathrm{W}(0.1 \mathrm{M} \mathrm{HCl}$ aqueous solution containing 5 $\mathrm{mM} \mathrm{H}_{2} \mathrm{PdCl}_{4}$ and $5 \mathrm{mM} \mathrm{HAuCl}_{4}, \mathrm{pH}=1.4$ ) was gently pipetted onto $0.2 \mathrm{~g}$ of RAIL $\left[\mathrm{FcMDDA}^{+}\right]\left[\mathrm{TFPB}^{-}\right]$(synthesis method is in Supporting Information) in a $3 \mathrm{~mL}$ glass bottle, in order to form the RAIL/W interface. Then the system was kept at $70{ }^{\circ} \mathrm{C}$ for $24 \mathrm{~h}$ in a thermostatic bath. After the reaction, $\mathrm{W}$ was removed by a pipette first, and then black deposits left on the RAIL surface was washed by adding a mixed solvent (50:50 vol \%) of methanol and dichloromethane (DCM) followed by centrifugation and removal of the supernatant liquid. This washing-centrifugation procedure was repeated for 10 times.

\subsection{Evaluation of electrocatalytic activity}


An electrochemical working station equipped with a three-electrode system was used to carry out all the electrochemical measurements at room temperature, where the working electrode was a catalyst-loaded glassy carbon electrode (GCE), the reference electrode was the saturated calomel electrode (SCE), and the counter electrode was a Pt foil.

(1) Loading catalysts on GCE

A $3 \mathrm{~mm}$ diameter GCE with a geometric area of $0.071 \mathrm{~cm}^{2}$ was polished with $\mathrm{Al}_{2} \mathrm{O}_{3}$ particle suspension and then dried at room temperature in air after rinsing with Milli-Q water. $10 \mu \mathrm{L}$ of Au@Pd BNFs or $10 \mu \mathrm{L}$ Pd/C (Sigma-Aldrich, Palladium on activated charcoal, $10 \%$ Pd basis) dispersed ethanol solution $(1 \mathrm{mg} / \mathrm{mL})$ were dropcasted on the GCE surface. The Au@Pd BNFs or Pd/C catalyst loaded GCE was covered with $5 \mu \mathrm{L}$ of $0.05 \mathrm{wt} \%$ Nafion/ethanol solution for protection.

(2) Electrocatalytic measurements of catalysts

Cyclic voltammograms (CVs) in a $1 \mathrm{M} \mathrm{NaOH}$ solution were recorded to evaluate the electrochemically active surface area (EASA) of Au@Pd BNFs or Pd/C catalysts, by integrating the current at the PdO reduction region in the cathodic scan, where a value of $405 \mu \mathrm{C} / \mathrm{cm}^{2}$ was used for the reduction of $\mathrm{PdO}$ as previously reported ${ }^{[60]}$. The catalytic performance towards EOR for the catalysts was assessed by the CV and chronoamperometry (CA) measurements in a $1 \mathrm{M} \mathrm{NaOH}+1 \mathrm{M}$ ethanol solution. All solutions were purged with $\mathrm{N}_{2}$ before measurements.

\section{Results and discussion}

The morphology of the prepared Au@Pd BNFs was investigated by scanning electron microscope (SEM) and energy dispersive x-ray spectrometer (EDX) 
measurements performed simultaneously, and shown in Fig. 1a and 1b. Fig.1a reveals that the deposit consists of highly flexible and uniform 1D nanofibers with a diameter of 60 100 nm and a length of several tens micrometers. They formed dense and complex networks by intertwining with each other rather than aggregation. The EDX mappings (Fig. 1b) showing the element distributions for Pd and Au as well as the overlap, are identical to the morphology of the corresponding SEM image. The average atomic ratio of $\mathrm{Au}$ to $\mathrm{Pd}$ was $1: 1.2$, which is close to $1: 1$, the initial concentration ratio of $\mathrm{AuCl}_{4}{ }^{-}$to $\mathrm{PdCl}_{4}{ }^{2-}$ in $\mathrm{W}$. The lower Au content estimated from EDX probably results from fewer electrons penetrating into the Au core center. The overlapped EDX mapping for Au and Pd (Fig. 1b) implies that the structure of BNFs is Au core and Pd shell, which will be clearly demonstrated by the results at several different reaction times shown below. The results illustrated that the nanofibers are made of Pd and Au. Transmission electron microscopy (TEM) was also performed to verify the core-shell structure and the TEM image is shown in Fig. 1c. It can be clearly observed that each nanofiber contains a dark center (the Au core) and a gray shell with a jagged shape which consists of numerous small particles (the Pd shell), confirming the formation of the core-shell structure. X-ray diffraction (XRD) analysis was employed to identify the phase of the prepared nanofibers. The XRD pattern is shown in Fig. 1d with standard XRD patterns of pure Au and Pd as the references. The XRD pattern of the prepared nanofibers exhibits typical diffraction peaks of $\mathrm{Au}$ and $\mathrm{Pd}$, indicating the successful formation of bimetallic core-shell nanostructures.

To investigate the growth mechanism of Au@Pd BNFs, the synthesis was terminated at different reaction times $(t=1,4,8,12$, and $24 \mathrm{~h})$ and the corresponding SEM images and EDX mappings are shown in Fig. S1-S5. At $t=1 \mathrm{~h}$ (Fig. S1), short nanofibers were found as the deposit. EDX mapping manifests that no Pd was detected except for $\mathrm{Au}$, demonstrating that only Au nanofibers were preferentially formed in 
the initial $1 \mathrm{~h}$. In our previous studies, we have revealed that the formation of $1 \mathrm{D}$ nanostructures can be attributed to the synergetic effect of the high viscosity ${ }^{[42]}$ of IL and IL itself in the IL/W interfacial system ${ }^{[31-34]}$. Hence, in the present system, the same factors have induced the formation of Au nanofibers. For $t=4 \mathrm{~h}$ (Fig. S2), Pd slightly appeared with inhomogeneous distribution at some regions where Au was intensely detected, indicating that Pd just started to grow on the surface of Au at $4 \mathrm{~h}$. Such a phenomenon may be attributed to the high viscosity of the RAIL and therefore low diffusion rate of $\mathrm{PdCl}_{4}{ }^{2-}$ on the RAIL side of RAIL $\mid \mathrm{W}$ interface. For $t=8 \mathrm{~h}$ (Fig. S3) and $t=12 \mathrm{~h}$ (Fig. S4), EDX mappings display Pd distribution at the Au detected regions with gradually improved uniformity, which seems to result from the comparatively more uniform distribution of $\mathrm{PdCl}_{4}{ }^{2-}$ for a longer time. At the final stage at $t=24 \mathrm{~h}$ (Fig. S5), EDX analysis detected the uniform distribution of Au and $\mathrm{Pd}$ at the same analytic field which is in accordance with the morphology of nanofibers in the corresponding SEM image.

UV-vis spectra were measured for the $\mathrm{W}$ from above-terminated synthesis at different reaction times $(t=1,4,8,12$, and $24 \mathrm{~h})$ and the results are shown in Fig. 2. UV-vis spectra for the standard $\mathrm{HAuCl}_{4}(0.5 \mathrm{mM}$ in $\mathrm{HCl} 0.1 \mathrm{M})$ and $\mathrm{H}_{2} \mathrm{PdCl}_{4}(0.5 \mathrm{mM}$ in $\mathrm{HCl} 0.1 \mathrm{M}$ ) solutions were also measured for comparison, showing the characteristic absorption peaks at $313 \mathrm{~nm}$ for $\mathrm{AuCl}_{4}^{-}$and $426 \mathrm{~nm}$ for $\mathrm{PdCl}_{4}{ }^{2-}$ (Fig. S6). Their corresponding molar absorptivity $\varepsilon$ at $313 \mathrm{~nm}$ and $426 \mathrm{~nm}$ were calculated, in order to estimate the concentration of each component in $\mathrm{W}$ from the overlapped absorbance (the detailed calculation and the results are in Supporting Information). After $1 \mathrm{~h}$ reaction (Fig. 2), the concentration of $\mathrm{AuCl}_{4}^{-}$in $\mathrm{W}$ decreases around $90 \%$ whereas the concentration of $\mathrm{PdCl}_{4}{ }^{2-}$ in $\mathrm{W}$ remains unchanged compared with their initial concentrations before the reaction $(t=0)$. Moreover, the absorbance of the peaks $(t=1,4,8,12 \mathrm{~h})$ at $426 \mathrm{~nm}$ continuously decreased until $t=12 \mathrm{~h}$. At $t=24 \mathrm{~h}$, 
the characteristic absorption of $\mathrm{AuCl}_{4}{ }^{-}$and $\mathrm{PdCl}_{4}{ }^{2-}$ thoroughly disappeared, indicating that the metal precursors were fully reacted and thus the average molar ratio of $\mathrm{Au}$ and Pd for the BNFs is estimated as 1: 1 . The UV-vis spectra revealed that $\mathrm{AuCl}_{4}{ }^{-}$is largely consumed in the first $1 \mathrm{~h}$ and after that $\mathrm{PdCl}_{4}{ }^{2-}$ starts to be reduced until $\mathrm{PdCl}_{4}{ }^{2-}$ in W are completely depleted. The above results are consistent with the EDX analysis, confirming that $\mathrm{AuCl}_{4}{ }^{-}$is reduced prior to $\mathrm{PdCl}_{4}{ }^{2-}$ and that the growth of Au@Pd BNFs proceeds segmentally.

To demonstrate the role of $\mathrm{Au}$ as the core for the growth of Pd, the initial concentration ratio of $\mathrm{AuCl}_{4}{ }^{-}$to $\mathrm{PdCl}_{4}{ }^{2-}$ was varied from the typical 1:1 to 1:0, 1:5, 1:10, and 0:1. For the 1:0 ratio, i.e., in the absence of $\mathrm{PdCl}_{4}{ }^{2-}$, the SEM and EDX images in Fig. S7 show that Au nanofibers are formed even when only $\mathrm{AuCl}_{4}{ }^{-}$is added. The results for the 1:1 ratio were already shown in Fig. 1; long Au@Pd nanofibers are obtained. However, when the ratio was varied toward higher concentrations of $\mathrm{PdCl}_{4}{ }^{2-}$ such as 1:5 (Fig. S8) and 1:10 (Fig. S9), nanofibers apparently become shorter. Finally, when the ratio reaches 0:1 (Fig. S10), in the absence of $\mathrm{AuCl}_{4}^{-}$, a segmented nanostructure was obtained, which is comprised of particles on the top, short nanofibers in the middle and firm sheets on the bottom. The formation mechanism of such Pd nanostructures was discussed in our previous report ${ }^{[34]}$. The above results manifest that and Pd cannot form 1D structure individually in the present system. Au nanofibers are first formed without any interference from Pd, and then play a role of 1D nanoframe for the growth of the Pd shell. This may be explained by the large difference of their standard reduction potentials $\left(1.002 \mathrm{~V}\right.$ for $\mathrm{AuCl}_{4}{ }^{-}$, and $0.591 \mathrm{~V}$ for $\left.\mathrm{PdCl}_{4}{ }^{2-}\right)^{[43]}$, which will be significant enough for $\mathrm{AuCl}_{4}{ }^{-}$and $\mathrm{PdCl}_{4}{ }^{2-}$ to be reduced sequentially.

It is worth noting that, in Fig.2, a small peak at $627 \mathrm{~nm}$ arises since $t=4 \mathrm{~h}$. This can be attributed to the oxidized divalent cation FcMDDA ${ }^{2+[34]}$, which is generated 
via the electron transfer (ET) reactions between the metal precursors and redox-active cations FcMDDA $^{+}$(we measured the standard redox potential of $\mathrm{FcMDDA}^{2+} / \mathrm{FcMDDA}^{+}$which is $0.26 \mathrm{~V}$ vs. $\mathrm{Fc}^{+} / \mathrm{Fc}$, in acetonitrile containing $0.1 \mathrm{M}$ tetraethylammonium perchlorate at $298 \mathrm{~K})$ :

$$
\begin{aligned}
& 3 \mathrm{FcMDDA}^{+}(\mathrm{RAIL})+\mathrm{AuCl}_{4}^{-}(\mathrm{W}) \rightarrow 3 \mathrm{FcMDDA}^{2+}(\mathrm{RAIL})+\mathrm{Au}+4 \mathrm{Cl}^{-}(\mathrm{W})(1) \\
& 2 \mathrm{FcMDDA}^{+}(\mathrm{RAIL})+\mathrm{PdCl}_{4}^{2-}(\mathrm{W}) \rightarrow 2 \mathrm{FcMDDA}^{2+}(\mathrm{RAIL})+\mathrm{Pd}+4 \mathrm{Cl}^{-}(\mathrm{W})(2)
\end{aligned}
$$

The above ET reactions were investigated by an electron conductor separating oil-water (ECSOW) ${ }^{[44]}$ system, where the RAIL and W are separated but electrically connected by a metal wire so that only ET processes are measured, and the results are shown in figure S12. The midpoint potentials $\left(E_{\text {mid }}\right)$ of the ET reactions are marked as the blue and red dashed lines, respectively, showing $420 \mathrm{mV}$ more negative $E_{\text {mid }}$ for Au (eqn (1)) compared with Pd (eqn (2)). The results clearly manifest that the redox reaction of $\mathrm{AuCl}_{4}^{-}$with $\mathrm{FcMDDA}^{+}$is significantly favored to take place compared with that of $\mathrm{PdCl}_{4}{ }^{2-}$.

The UV spectra in Fig. 2 demonstrate that FcMDDA ${ }^{2+}$ has existed in $\mathrm{W}$ after $4 \mathrm{~h}$ reaction, whereas it has not been found in the $\mathrm{W}$ within $t=1 \mathrm{~h}$. $\mathrm{FcMDDA}^{2+}$ in $\mathrm{W}$ is the result of the ion transfer (IT) of FcMDDA ${ }^{2+}$ from RAIL to W (eqn 3):

$$
\mathrm{FcMDDA}^{2+}(\mathrm{RAIL}) \rightarrow \mathrm{FcMDDA}^{2+}(\mathrm{W})
$$

In other words, the IT of FcMDDA ${ }^{2+}$ does not occur when $\mathrm{AuCl}_{4}{ }^{-}$is under reduction, but takes place along with the reduction of $\mathrm{PdCl}_{4}{ }^{2-}$. This may be due to more negative $E_{\text {mid }}$ for the IT of $\mathrm{PdCl}_{4}{ }^{2-}$ and $\mathrm{FcMDDA}^{2+}$ than $\mathrm{AuCl}_{4}{ }^{-}$(see below for further discussion). However, the peak of FcMDDA ${ }^{2+}$ disappeared at $t=24 \mathrm{~h}$, probably due 
to the decomposition of the FcMDDA ${ }^{2+}$ ions in W. A FcMDDA ${ }^{2+}$ solution was prepared and kept at $70^{\circ} \mathrm{C}$ for 3 days in order to verify the stability of FcMDDA ${ }^{2+}$ ions in W. As shown in Fig. S13, the color of the solution changed from dark green, which originates from the FcMDDA ${ }^{2+}$, to colorless, and some brown-red precipitates were found at the bottom of the solution. This result indicates that FcMDDA ${ }^{2+}$ is unstable in $\mathrm{W}$ and easy to be decomposed. Some references also mentioned that ferrocenium ions are unstable in $\mathrm{W}^{[45,46]}$. In previous studies ${ }^{[31-34]}$ we have revealed the fact that for the IL/W interfacial system, because of the separation of reductant and oxidant in IL and W, the ET reaction cannot occur independently on account of the unbalanced charges in each liquid phase as was presented in eqns 1 and 2, and other charge transfer process with the opposite direction of ET is required to be coupled with ET to neutralize the excess charges brought by ET. A confirmed conclusion drawn from the previous studies ${ }^{[31-34]}$ is that the IT of $\mathrm{AuCl}_{4}{ }^{-}$and $\mathrm{PtCl}_{4}{ }^{2-}$ from W to IL play roles not only for coupling with ET, but also for providing sources for the growth of Au and Pt nanofibers in IL. Similarly, the IT of $\mathrm{AuCl}_{4}{ }^{-}$and $\mathrm{PdCl}_{4}{ }^{2-}$ from W to RAIL can also be expected to play the same roles in the present case (eqns 4 and 5):

$$
\begin{aligned}
\mathrm{AuCl}_{4}^{-}(\mathrm{W}) & \rightarrow \mathrm{AuCl}_{4}^{-}(\mathrm{RAIL}) \\
\mathrm{PdCl}_{4}{ }^{2-}(\mathrm{W}) & \rightarrow \mathrm{PdCl}_{4}{ }^{2-}(\mathrm{RAIL})
\end{aligned}
$$

For the RAIL|W system, it is impossible to measure individual IT processes of eqns (4) and (5) since in the presence of $\mathrm{AuCl}_{4}{ }^{-}$and $\mathrm{PdCl}_{4}{ }^{2-}$ in $\mathrm{W}$, the ET processes of eqns (1) and (2) also occur, superimpose in the current, and interfere the IT measurements. Therefore, a redox-inactive IL trihexyltetradecylphosphonium tetrakis(3,5-bis(trifluoromethyl)phenyl)borate ([THTDP][TFPB]) was adopted as the substitution for the RAIL, in order to investigate IT processes of eqns (3), (4) and (5). 
As illustrated in Fig. S14, $E_{\text {mid }}$ for the IT of $\mathrm{AuCl}_{4}{ }^{-}$is $205 \mathrm{mV}$ more positive than that of $\mathrm{PdCl}_{4}{ }^{2-}$, indicating a more thermodynamically favored IT behavior from W to IL for $\mathrm{AuCl}_{4}^{-}$than $\mathrm{PdCl}_{4}{ }^{2-}$. This relationship is expected to be applied to the RAIL/W system. Since both the IT and ET reactions for $\mathrm{AuCl}_{4}{ }^{-}$is more favored than the counterparts for $\mathrm{PdCl}_{4}{ }^{2-}$, $\mathrm{AuCl}_{4}{ }^{-}$will be reduced prior to $\mathrm{PdCl}_{4}{ }^{2-}$.

Based on the above evidence and discussion, the interfacial reaction mechanism for the formation of Au@Pd BNFs can be inferred as two-stage reactions, as shown in the schematic (Fig. 3). For the first stage of the reduction of $\mathrm{AuCl}_{4}{ }^{-}$, the ET of eqn (1) is coupled with the IT of eqn (4) and preferentially proceed as described in the total reaction (eqn (6)):

$$
\begin{gathered}
3 \mathrm{FcMDDA}^{+}(\mathrm{RAIL})+4 \mathrm{AuCl}_{4}^{-}(\mathrm{W}) \rightarrow 3 \mathrm{FcMDDA}^{2+}(\mathrm{RAIL})+\mathrm{Au}+4 \mathrm{Cl}^{-}(\mathrm{W})+ \\
3 \mathrm{AuCl}_{4}^{-}(\mathrm{RAIL})(6)
\end{gathered}
$$

The reduction of $\mathrm{AuCl}_{4}{ }^{-}$occurs first as it is thermodynamically favored and only takes $1 \mathrm{~h}$ to deplete over $90 \%$ of $\mathrm{AuCl}_{4}^{-}$in $\mathrm{W}$. After that, the second stage of the reduction of $\mathrm{PdCl}_{4}{ }^{2-}$ starts to proceed. The ET of eqn (2) is primarily coupled with the IT of eqn (3) and in addition, also partly coupled with the IT of eqn (5) because of the similar IT potentials. The total reaction can be qualitatively described in the eqn (7):

$$
\begin{gathered}
2 \mathrm{FcMDDA}^{+}(\mathrm{RAIL})+(2-\alpha) \mathrm{PdCl}_{4}{ }^{2-}(\mathrm{W}) \rightarrow(2-\alpha) \mathrm{FcMDDA}^{2+}(\mathrm{RAIL})+\mathrm{Pd}+4 \mathrm{Cl}^{-} \\
(\mathrm{W})+(1-\alpha) \mathrm{PdCl}_{4}{ }^{2-}(\mathrm{RAIL})+\alpha \mathrm{FcMDDA}^{2+}(\mathrm{W})(7)
\end{gathered}
$$

where $\alpha$ is the faction of the IT for the FcMDDA ${ }^{2+}$ in the total IT processes (eqn (3) and (5)). The two-stage reactions can spontaneously occur in order without interfering each other due to both the large ET and IT potential differences. 
In order to authenticate the role of RAIL/W interfacial system, the synthesis procedure was modified as follows: (1) firstly, the synthesis was conducted as the same as the typical one at $70{ }^{\circ} \mathrm{C}$ for $1 \mathrm{~h}$ for the sake of the favoring formation of $\mathrm{Au}$ nanofibers as the template; (2) at $t=1 \mathrm{~h}, 5 \mathrm{~mL}$ methanol was added for altering the two-phase system into one phase, and the mixture was reacted at $70{ }^{\circ} \mathrm{C}$ for the next $23 \mathrm{~h}$ under stirring. Fig. S15 show the SEM image of the obtained nanostructures, which are still nanofibers. However, they are unlike the Au@Pd BNFs prepared at the RAIL/W interface (Fig. 1 and S7); the obtained product is severely agglomerated together rather than forming network structures. This difference confirms the indispensable role of the RAIL $\mid \mathrm{W}$ interface, restraining the agglomeration of nanofibers growing inside RAIL. Considering that a large amount of RAIL-ions still exist in the one-phase mixture, the remarkable protection effect for nanofibers from agglomeration is unlikely to be caused by the selective adsorption of RAIL-ions on the metal surface like other capping agents, but the rigid multilayering structures formed by the IL-ions at the interface may prevent the agglomeration of metal nanostructures. Such kind of protection from agglomeration by IL-ions is in contrast to the conventional methods using capping agents. EDX analysis was performed for the obtained nanofibers and the results are shown in Fig. S15. Unexpectedly, the EDX mappings show that $\mathrm{Au}$ is prevailingly distributed whereas the $\mathrm{Pd}$ is seldom distributed at the analytic field, and the average atomic ratio for Au and Pd is 96:4. That is, the reduction and growth of Pd on the surface of Au nanofibers were basically failed to proceed in the one-phase mixture. This may be explained by the particularity of the RAIL, which can be regarded as a dense phase with a significantly high concentration of reducing agent, greatly facilitating the reduction of the transferred $\mathrm{PdCl}_{4}{ }^{2-}$ ions on the RAIL side of the RAIL/W interface. The above results reveal the crucial roles of the RAIL/W interface played in the system. 
The catalytic activity of Au@Pd BNFs for direct ethanol fuel cell was evaluated by measuring the electrocatalytic activity toward EOR in an alkaline media, and the commercial Pd/C catalyst was also measured for comparison. CVs for Au@Pd BNFs and $\mathrm{Pd} / \mathrm{C}$ catalysts in a $\mathrm{N}_{2}$-purged $1 \mathrm{M} \mathrm{NaOH}$ solution were shown in Fig. 4a. The EASA of the catalysts was evaluated from the charge of the reduction current of Pd oxide, where the loading amount of Pd for Au@Pd BNFs was estimated by taking the average atomic ratio for Au and Pd is 1: 1. The EASA of Au@Pd BNFs was found to be $7 \mathrm{~m}^{2} / \mathrm{g}$, which is smaller than that of the Pd/C catalyst $\left(62 \mathrm{~m}^{2} / \mathrm{g}\right)$. The relatively lower EASA for Au@Pd BNFs can be attributed to the different size and structure between 1D nanofiber and 0D particle. The CVs for Au@Pd BNFs and Pd/C catalysts were carried out in a $1 \mathrm{M} \mathrm{NaOH}+1 \mathrm{M}$ ethanol solution at the scan rate of $50 \mathrm{mV} / \mathrm{s}$ in order to evaluate their catalytic performance toward EOR, and the current density was normalized on the basis of the EASA of Pd (Fig. 4b). Two oxidation peaks can be clearly discerned, which corresponds to the oxidation of ethanol in the forward scan $\left(I_{\mathrm{f}}\right)$ and back scan $\left(I_{\mathrm{b}}\right)^{[47]}$, The Au@Pd BNFs present much higher specific activity with Pd/C toward EOR due to $0.1 \mathrm{~V}$ negative onset potential $(-0.7 \mathrm{~V}$ for $\mathrm{Au} @ \mathrm{Pd}$ BNFs and $-0.6 \mathrm{~V}$ for $\mathrm{Pd} / \mathrm{C})$ and 3.6 times higher $I_{\mathrm{f}}$ compared with that of $\mathrm{Pd} / \mathrm{C}(6.4$ $\mathrm{mA} / \mathrm{cm}^{2}$ for Au@Pd BNFs and $1.8 \mathrm{~mA} / \mathrm{cm}^{2}$ for $\mathrm{Pd} / \mathrm{C}$ ). Furthermore, chronoamperometry (CA) measurements were performed in a $1 \mathrm{M} \mathrm{NaOH}+1 \mathrm{M}$ ethanol solution at a constant potential of $-0.3 \mathrm{~V}$, and the results are shown in Fig. 4c. The currents for the two catalysts both decayed rapidly at the initial period, which can be ascribed to surface poisoning by the accumulation of adsorbed reaction intermediates on the active sites, and reached a pseudo-steady state at $2000 \mathrm{~s}\left(I_{\mathrm{s}}\right)$. Au@Pd BNFs exhibit 5.7 times higher $I_{\mathrm{s}}\left(0.40 \mathrm{~mA} / \mathrm{cm}^{2}\right)$ compared with that of $\mathrm{Pd} / \mathrm{C}$ $\left(0.07 \mathrm{~mA} / \mathrm{cm}^{2}\right)$, illustrating much better durability for EOR in alkaline media. The excellent electrocatalytic performance of Au@Pd BNFs can be explained from the 
following two reasons. First, compared with Pd/C, Au@Pd BNFs possess more effective electron conduction pathways by depositing Pd nanoparticles on the 1D structure of Au nanofibers, which contributes to improving the electrocatalytic activity. Second, the introduction of Au for Pd in the bimetallic nanostructures also plays an important role. This may be explained by the $d$-band center theory, which was first proposed by Nørskov and co-workers ${ }^{[48,49]}$. It was reported that catalytic ability of metal is strongly related to its $d$-band center (which is relative to the Fermi level) ${ }^{[49]}$, where a higher $d$-band center induces stronger chemisorption of species on the metal surface. According to reference data, Au has larger lattice size than Pd. The introduction of $\mathrm{Au}$ in Pd nanostructure causes a positive shift for the $d$-band center ${ }^{[50-}$ 52], resulting in a higher adsorption rate of the intermediates, especially for $\mathrm{OH}^{-}$, which is confirmed to be crucial for stripping off the adsorbed intermediate species on the metal surface ${ }^{[50]}$. Thereby, the intermediate species which could cause the surface-poisoning by blocking the active sites on the catalyst will be removed more efficiently because of the stronger chemisorption of $\mathrm{OH}^{-}$. The TEM image of Fig. 1(c) reveals that Au@Pd BNFs are composed of a large amount Pd nanoparticles covering at the Au nanofiber surfaces as the shell. It can be observed that such Pd shell made of nanoparticles presents a jagged shape with non-uniform thickness from the minimum of several nanometers to the maximum of around $40 \mathrm{~nm}$. Some studies reported that the enhancement towards EOR activity of Au for Pd only works for thin Pd shell nanostructures ${ }^{[53]}$. With increasing the thickness of the Pd shell, the lattice strain effect, which results in the positive shifted $d$-band center and the enhanced bonding strength of adsorbate, will be gradually weakened until disappeared ${ }^{[53]}$. Therefore, in thin-shell regions of Pd, the activity is greatly improved, but for the thick-shell regions of Pd, such kind of effect is restrained.

The stability of Au@Pd BNFs and Pd/C was checked by performing continuous 
100-time CV cycles in a $1 \mathrm{M} \mathrm{NaOH}$ and $1 \mathrm{M} \mathrm{NaOH}+1 \mathrm{M}$ ethanol solution at 50 $\mathrm{mV} / \mathrm{s}$ and the results are shown in Fig. S16. The comparison of the EASA and the catalytic ability of Au@Pd BNFs between the $1^{\text {st }}$ and $100^{\text {th }}$ cycles are shown in Fig. 5(a) and 5(b), respectively. In Fig. 5(a), an apparently increased current peak of the reduction of PdO for Au@Pd BNFs after 100-time cycles can be observed, and the corresponding EASA was estimated to be increased by 2.7 times $\left(7 \mathrm{~m}^{2} / \mathrm{g}\right.$ for the $1^{\text {st }}$ and $19 \mathrm{~m}^{2} / \mathrm{g}$ for the $100^{\text {th }}$ ). In Fig. 5(b), If of Au@Pd BNFs increases by 1.4 time (420 $\mathrm{mA} / \mathrm{mg}$ for the $1^{\text {st }}$ and $600 \mathrm{~mA} / \mathrm{mg}$ for the $100^{\text {th }}$ ), indicating an enhanced catalytic ability after 100-time cycles. The above results differ from the conventional phenomenon; the long-term cycling causes a decrease in the EASA of catalyst due to the dissolution of metal, consequently degrading the catalytic ability. In fact, this phenomenon was found for Pd/C, where the EASA decreased by 2.8 times $\left(62 \mathrm{~m}^{2} / \mathrm{g}\right.$ for the $1^{\text {st }}$ and $22 \mathrm{~m}^{2} / \mathrm{g}$ for the $100^{\text {th }}$, Fig. S17 (a)) and $I_{\mathrm{f}}$ decreased by 1.2 time (1150 $\mathrm{mA} / \mathrm{mg}$ for the $1^{\text {st }}$ and $980 \mathrm{~mA} / \mathrm{mg}$ for the $100^{\text {th }}$, Fig. S17 (b)) after 100-time cycles. Unlike the ordinary cases where catalysts maintain high stability by resisting degradation, Au@Pd BNFs show even higher catalytic ability after 100-time cycles. Such unusual enhancement by cycling times is rarely seen in the literature ${ }^{[53,54]}$. Ksar et al. reported an improved EOR activity of Au-Pd core-shell nanoparticles after 200time cycles, which may be attributed to the high defect density of Pd in the particle shell ${ }^{[54]}$. Brodsky et al. proposed surface metal migration theory to explain the enhanced catalytic activity of Au-Pd core-shell nanoparticles with thin Pd shell after 500-time cycles, where Au gradually migrates from the core to Pd shell during cycling and thus the EOR activity is increased by the strengthened lattice strain effect ${ }^{[53]}$. In the other most cases, Au-Pd bimetallic nanostructures display high stability with a slight decrease of activity during long-term cycling ${ }^{[50,55-58]}$, rather than the increase observed for the Au-Pd core-shell nanoparticles ${ }^{[53,54]}$. For the present case of Au-Pd 
BNFs, the cathodic and anodic peaks between around $-0.8 \mathrm{~V}$ and $-0.6 \mathrm{~V}$ (Fig. 5(a)) corresponding to the adsorption/desorption of hydrogen at Pd surface ${ }^{[59,60]}$, gradually increased as shown in Fig. S16(a), and both significantly enhanced in the $100^{\text {th }}$ cycle compared with the $1^{\text {st }}$ cycle, implying a promoted adsorption behavior and increased EASA by cycling over 100 times. In contrast, for Pd/C, the adsorption/desorption peaks of hydrogen (between around $-0.8 \mathrm{~V}$ and $-0.5 \mathrm{~V}$ ) successively decreased (Fig. S16(c), Fig. S17(a)) during the 100-time cycles. On the other hand, the broad anodic peak associated with the adsorption of $\mathrm{OH}^{-}$on Pd surface followed by the formation of PdO layer (starts from around $-0.3 \mathrm{~V}$ ) is greatly enhanced for Au-Pd BNFs after 100-time cycles, corresponding to the enhanced reduction peak of PdO at the cathodic scan. But for Pd/C, both weakened oxidation and reduction peaks of Pd indicates a decrease of $\mathrm{OH}^{-}$adsorption and EASA after 100-time cycles. The distinct contrast between Au@Pd BNFs and Pd/C may result from the different chemical composition of the two catalysts. The dissolution of Pd metal usually occurs under electrochemical measurements ${ }^{[50,57,61]}$, which could be the reason for the reduced EASA of $\mathrm{Pd} / \mathrm{C}$. However, for the case of Au@Pd BNFs, the partial dissolution of Pd seems to have enhanced its catalytic ability. TEM measurement was performed for the Au-Pd BNFs before and after the EOR cycling for 100 times, and the results are shown in Fig. 5(d) and (e) along with the schematics at the right-bottom corner. It can be observed that the Pd nanoparticles constituting Pd shell before the EOR cycles (Fig. 5(d)) have transformed to smaller nanoparticles (Fig. 5(e)) after EOR cycles, increasing the area of thin-shell regions and accordingly the EASA. Since the lattice strain effect is limited at the thick-shell regions of Pd in the jagged shape, such transformation not only increases the EASA but also strengthens the lattice strain effect. The improved adsorption behavior for both hydrogen and $\mathrm{OH}^{-}$in Fig. S16(a) reflects an enhanced lattice strain effect, which results from the increased thin-shell regions. Besides, the 
1D nanostructures of Au-Pd BNFs with a length of several tens of micrometers can keep relatively stable compared with the OD structures of nanoparticles. For the EOR measurements, the degraded catalytic ability for $\mathrm{Pd} / \mathrm{C}$ catalyst in the $100^{\text {th }}$ cycle can be attributed to the decreased EASA due to the dissolution and the aggregation of Pd during cycling; Au@Pd BNFs show an enhanced catalytic ability for the $100^{\text {th }}$ cycle, which could be a synergetic effect of the increased EASA and the promoted chemisorption of $\mathrm{OH}^{-}$on metal surface by thinning the Pd shell. A plot of $I_{\mathrm{f}}$ in Fig. S16(b) against cycle number was drawn in Fig. 5(c) to display the change of the catalytic ability of Au@Pd BNFs during 100-time cycles. It can be seen that for the first 35 cycles, $I_{\mathrm{f}}$ increases rapidly, and then remains relatively stable at the maximum value for the next 65 cycles, indicating the catalytic ability of Au@Pd BNFs not only has been improved, but also remains high stability during long-term cycling.

\section{Conclusions}

We have prepared Au@Pd core-shell BNFs by an automatically sequential reduction of $\mathrm{AuCl}_{4}{ }^{-}$and $\mathrm{PdCl}_{4}{ }^{2-}$ at the $\mathrm{RAIL} \mid \mathrm{W}$ interface. $\mathrm{AuCl}_{4}{ }^{-}$is reduced first and spontaneously form Au nanofibers which become the core for the following growth of Pd. The detailed reaction and growth mechanism was revealed and discussed. The RAIL was confirmed to actively play multiple roles in the RAIL/W interfacial system, not only by acting as the reducing agent for metal precursors and the liquid substrate, but also by facilitating the reduction of $\mathrm{Pd}$ as well as preventing the agglomeration of nanofibers. The prepared Au@Pd BNFs not only show higher catalytic performance toward EOR than a commercial $\mathrm{Pd} / \mathrm{C}$ catalyst, but also present an even improved catalytic ability and high stability during 100-time cycles. 


\section{Acknowledgement}

This work was partly supported by JSPS KAKENHI (No. 18K05171) and TEPCO Memorial Foundation.

\section{References}

[1] X. Huang and N. Zheng, J. Am. Chem. Soc., One-pot, high-yield synthesis of 5-fold twinned Pd nanowires and nanorods, 131 (2009) 4602-4603.

[2] S. E. Skrabalak, L. Au, X. Li, Y. Xia, Facile synthesis of Ag nanocubes and Au nanocages, Nat. Protoc., 2 (2007) 2182-2190.

[3] R. Bardhan, N. K. Grady, J. R. Cole, A. Joshi, N. J. Halas, Fluorescence enhancement by Au nanostructures: nanoshells and nanorods, ACS. Nano, 3 (2009) 744-752.

[4] M. Sanles-Sobrido, M. A. Correa-Duarte, S. Carregal-Romero, B. Rodríguez-González, R. A. Álvarez-Puebla, P. Hervés, L. M. Liz- Marzán, Highly Catalytic Single-Crystal Dendritic Pt Nanostructures Supported on Carbon Nanotubes, Chem. Mater., 21 (2009) $1531-1535$.

[5] C. Wang, Y. Hu, C.M. Lieber, S. Sun, Ultrathin Au nanowires and their transport properties, J. Am. Chem. Soc., 130 (2008) 8902-8903.

[6] S. Sun, F. Jaouen, J.-P. Dodelet, Controlled growth of Pt nanowires on carbon nanospheres and their enhanced performance as electrocatalysts in PEM fuel cells, Adv. Mater., 20 (2008) 3900-3904.

[7] Y. Chang, M. L. Lye, H. C. Zeng, Large-scale synthesis of high-quality ultralong copper nanowires, Langmuir, 21 (2005) 3746-3748.

[8] Y. Bi, G. Lu, Facile synthesis of platinum nanofiber/nanotube junction structures at room temperature, Chem. Mater., 20 (2008) 1224-1226. 
[9] N. S. Hansen, D. Cho, Y. L. Joo, Metal Nanofibers with Highly Tunable Electrical and Magnetic Properties via Highly Loaded Water - Based Electrospinning, Small, 8 (2012) 1510-1514.

[10]H. Wu, L. Hu, M. W. Rowell, D. Kong, J. J. Cha, J. R. McDonough, J. Zhu, Y. Yang, M. D. McGehee, Y. Cui, Electrospun metal nanofiber webs as high-performance transparent electrode, Nano Lett., 10 (2010) 4242-4248.

[11] H. Zhu, H. Chen, J. Wang, Q. Li, Fabrication of Au nanotube arrays and their plasmonic properties, Nanoscale, 5 (2013) 3742-3746.

[12] X. Zhang, D. Dong, D. Li, T. Williams, H. Wang, P. A. Webley, Direct electrodeposition of Pt nanotube arrays and their enhanced electrocatalytic activities, Electrochem. Commun., 11 (2009) 190-193.

[13]M. Steinhart, Z. Jia, A. K. Schaper, R. B. Wehrspohn, U. Gösele, J. H. Wendorff, Adv. Mater., 15 (2003) 706-709.

[14]B. Y. Xia, H. B. Wu, Y. Yan, X. W. Lou, X. Wang, Palladium nanotubes with tailored wall morphologies, J. Am. Chem. Soc., 135 (2013) 9480-9485.

[15] S. E. Hunyadi, C. J. Murphy, Bimetallic silver-gold nanowires: fabrication and use in surface-enhanced Raman scattering, J. Mater. Chem., 16 (2006) 3929-3935.

[16]S. Guo, S. Dong, E. Wang, Ultralong Pt-on-Pd bimetallic nanowires with nanoporous surface: nanodendritic structure for enhanced electrocatalytic activity, Chem. Commun., 46 (2010) 1869-1871.

[17]N. L. Netzer, C. Qiu, Y. Zhang, C. Lin, L. Zhang, H. Fong, C. Jiang, Gold-silver bimetallic porous nanowires for surface-enhanced Raman scattering, Chem. Commun., 47 (2011) 9606-9608.

[18]C. Zhu, S. Guo, S. Dong, PdM (M= Pt, Au) Bimetallic Alloy Nanowires with Enhanced Electrocatalytic Activity for Electro - oxidation of Small Molecules, Adv. Mater., 24 (2012) 2326-2331. 
[19]W. Hong, J. Wang, E. Wang, Dendritic Au/Pt and Au/PtCu nanowires with enhanced electrocatalytic activity for methanol electrooxidation, Small, 10 (2014) 3262-3265.

[20]F. Cheng, X. Dai, H. Wang, S. P. Jiang, M. Zhang, C. Xu, Synergistic effect of Pd-Au bimetallic surfaces in Au-covered Pd nanowires studied for ethanol oxidation, Electrochimi. Acta, 55 (2010) 2295-2298.

[21]X. Gao, F. Lu, B. Dong, Y. Liu, Y. Gao, L. Zheng, Facile synthesis of gold and goldbased alloy nanowire networks using wormlike micelles as soft templates, Chem. Commun., 51 (2015) 843-846.

[22]W. Hong, J. Wang, E. Wang, Facile synthesis of $\mathrm{PtCu}$ nanowires with enhanced electrocatalytic activity, Nano Res., 8 (2015) 2308-2316.

[23]H. Wang, L. Qi, Controlled Synthesis of $\mathrm{Ag}_{2} \mathrm{~S}, \mathrm{Ag}_{2} \mathrm{Se}$, and Ag Nanofibers by Using a General Sacrificial Template and Their Application in Electronic Device Fabrication, Adv. Funct. Mater., 18 (2008) 1249-1256.

[24] J. Wang, D. Wang, N. S. Sobal, M. Giersig, M. Jiang, H. Mohwald, stepwise directing of nanocrystals to self-assemble at water/oil interfaces, Angew. Chem. Int. Ed. Engl. 45 (2006) 7963-7986.

[25] M. Brust, M. Walker, D. Bethell, D. J. Schiffrin, R. Whyman, Synthesis of thiolderivatised gold nanoparticles in a two-phase liquid-liquid system, J. Chem. Soc., Chem. Commun., 1994, 801-802.

[26]M. Platt, R. A. W. Dryfe, E. P. L. Roberts, Structural and electrochemical characterisation of Pt and Pd nanoparticles electrodeposited at the liquid/liquid interface, Electrochimi. Acta, 49 (2004) 3937-3945.

[27] M. Platt, R. A. W. Dryfe, Structural and electrochemical characterisation of Pt and Pd nanoparticles electrodeposited at the liquid/liquid interface: Part 2, Phys. Chem. Chem. Phys., 7 (2005) 1807-1814.

[28]K. Yao, W. Lu, X. Li, J. Wang, Tailoring the properties of aqueous-ionic liquid 
interfaces for tunable synthesis and self-assembly of ZnS nanoparticles, J. Mater. Chem. A, 2 (2014) 5140-5148.

[29] W. Lu, K. Yao, J. Wang, J. Yuan, Ionic liquids-water interfacial preparation of triangular Ag nanoplates and their shape-dependent antibacterial activity, J. Colloid Interface Sci., 437 (2015) 35-41.

[30]T. Soejima, N. Kimizuka, Ultrathin gold nanosheets formed by photoreduction at the ionic liquid/water interface, Chem. Lett., 34 (2005) 1234-1235.

[31]N. Nishi, T. Kakinami, T. Sakka, Dendritic nanofibers of gold formed by the electron transfer at the interface between water and a highly hydrophobic ionic liquid, Chem. Commun., 51 (2015) 13638-13641.

[32] N. Nishi, I. Yajima, K. Amano, T. Sakka, Janus-Type Gold/Polythiophene Composites Formed via Redox Reaction at the Ionic Liquid| Water Interface, Langmuir, 34 (2018) 2441-2447.

[33]Y. Zhang, N. Nishi, K. Amano, One-dimensional Pt nanofibers formed by the redox reaction at the ionic liquid| water interface, T. Sakka, Electrochim. Acta, 282 (2018) 886891.

[34]Y. Zhang, N. Nishi, T. Sakka, Template-free and spontaneous formation of vertically aligned Pd nanofiber arrays at the liquid-liquid interface between redox-active ionic liquid and water, ACS Appl. Mater. Interfaces, 26 (2019) 23731-23740.

[35] N. Nishi, T. Uruga, H. Tanida, T. Kakiuchi, Temperature dependence of multilayering at the free surface of ionic liquids probed by X-ray reflectivity measurements, Langmuir, 27 (2011) 7531-7536.

[36] N. Nishi, T. Uruga, H. Tanida, Potential dependent structure of an ionic liquid at ionic liquid/water interface probed by x-ray reflectivity measurements, J. Electroanal. Chem., 759 (2015) 129-136.

[37]R. Atkin, G. G. Warr, Structure in confined room-temperature ionic liquids, J. Phys. 
Chem. C, 111 (2007) 5162-5168.

[38]M. Mezger, H. Schröder, H. Reichert, S. Schramm, J. S. Okasinski, S. Schöder, V. Honkimäki, M. Deutsch, B. M. Ocko, J.; Ralston, M. Rohwerder, M. Stratmann, H. Dosch, Molecular layering of fluorinated ionic liquids at a charged sapphire (0001) surface, Science, 322 (2008) 424-428.

[39]N. Nishi, J. Uchiyashiki, Y. Ikeda, S. Katakura, T. Oda, M. Hino, N. L. Yamada, Potential-Dependent Structure of the Ionic Layer at the Electrode Interface of an Ionic Liquid Probed Using Neutron Reflectometry, J. Phys. Chem. C, 123 (2019) 9223-9230.

[40]J. Langmaier, A. Trojánek, Z. Samec, Electron transfer across the polarized interface between water and a hydrophobic redox-active ionic liquid, Electrochem. Commun., 12 (2010) 1333-1335.

[41]J. Langmaier, Z. Samec, Thermodynamic aspects of the electron transfer across the interface between water and a hydrophobic redox-active ionic liquid, Electrochim. Acta, 58 (2011) 606-613.

[42]F. Scholz, U. Hasse, Controlling the morphology of silver deposition at liquid| liquid interfaces: From nano-wires to super smooth films, Electrochem. Commun., 7 (2005) 541-546.

[43]A. J. Bard, R. Parsons, J. Jordan, Standard Potentials in Aqueous Solution, CRC Press, 1985, 287-365.

[44] H. Hotta, N. Akagi, T. Sugihara, S. Ichikawa, T. Osakai, Electron-conductor separating oil-water (ECSOW) system: a new strategy for characterizing electron-transfer processes at the oil/water interface, Electrochem. Commun., 4 (2002) 472-477.

[45]R. Wilson, D. J. Schiffrin, Chemiluminescence of Luminol Catalyzed by Electrochemically Oxidized Ferrocenes, Anal. Chem., 1996, 68 (1996) 1254-1257.

[46]A. Alkan, F. R. Wurm, Water-Soluble Metallocene-Containing Polymers, Macromol Rapid Commun., 37 (2016), 1482-1493. 
[47]N. V. Long, T. Duy Hien, T. Asaka, M. Ohtaki, M. Nogami, Synthesis and characterization of Pt-Pd alloy and core-shell bimetallic nanoparticles for direct methanol fuel cells (DMFCs): Enhanced electrocatalytic properties of well-shaped coreshell morphologies and nanostructures, Int. J. Hydrogen Energy, 36 (2011) 8478-8491.

[48]B. Hammer, J. K. Nørskov, Electronic factors determining the reactivity of metal surfaces, Surf. Sci., 343 (1995) 211-220.

[49] J. K. Nørskov, T. Bligaard, J. Rossmeisl, C. H. Christensen, Towards the computational design of solid catalysts, Nat. Chem., 1 (2009) 37-46.

[50]Q. Tan, C. Du, G. Yin, P. Zuo, X. Cheng, M. Chen, Highly efficient and stable nonplatinum anode catalyst with Au@Pd core-shell nanostructures for methanol electrooxidation, J. Catal., 295 (2012) 217-222.

[51]U. B. Demirci, Theoretical means for searching bimetallic alloys as anode electrocatalysts for direct liquid-feed fuel cells, J. Power Sources, 173(2007) 11-18.

[52]M. Mavrikakis, B. Hammer, J. K. Nørskov, Effect of strain on the reactivity of metal surfaces, Phys. Rev. Lett., 81 (1998) 2819-2822.

[53]C. N. Brodsky, A. P. Young, K.C. Ng, C. H. Kuo, and C. K. Tsung, Electrochemically induced surface metal migration in well-defined core-shell nanoparticles and its general influence on electrocatalytic reactions, ACS Nano, 8 (2014) 9368-9378.

[54]F. Ksar, L. Ramos, B. Keita, L. Nadjo, P. Beaunier, H. Remita, Bimetallic palladiumgold nanostructures: application in ethanol oxidation, Chem. Mater., 21 (2009) 36773683.

[55] J. B. Xu, T. S. Zhao, S. Y. Shen, Y. S. Li, Stabilization of the palladium electrocatalyst with alloyed gold for ethanol oxidation, Int. J. Hydrogen Energ., 35 (2010) 6490-6500.

[56]S. W. Kang, Y. W. Lee, M. Kim, J. W. Hong, S. W. Han, One - Pot Synthesis of Carbon - Supported Dendritic Pd-Au Nanoalloys for Electrocatalytic Ethanol Oxidation, Chem. Asian J., 6 (2011) 909-913. 
[57]G. Fu, Z. Liu, Y. Chen, J. Lin, Y. Tang, T. Lu, Synthesis and electrocatalytic activity of Au@ Pd core-shell nanothorns for the oxygen reduction reaction, Nano Res., 7 (2014) 1205-1214.

[58]C. Liu, X. Cai, J. Wang, J. Liu, A. Riese, Z. Chen, X. Sun and S.-D. Wang, One-step synthesis of AuPd alloy nanoparticles on graphene as a stable catalyst for ethanol electrooxidation, Int. J. Hydrogen Energ., 41 (2016) 13476-13484.

[59]Z. X. Liang, T. S. Zhao, J. B. Xu, L. D. Zhu, Mechanism study of the ethanol oxidation reaction on palladium in alkaline media, Electrochimi. Acta, 54 (2009) 2203-2208.

[60]R. N. Singh, A. Singh, Anindita, Electrocatalytic activity of binary and ternary composite films of Pd, MWCNT and Ni, Part II: methanol electrooxidation in $1 \mathrm{M} \mathrm{KOH}$, Int. J. Hydrogen Energ., 34 (2009) 2052-2057.

[61]E. Pizzutilo, S. J. Freakley, S. Geiger, C. Baldizzone, A. Mingers, G. J. Hutchings, K. J. J. Mayrhofer, S. Cherevko, Addressing stability challenges of using bimetallic electrocatalysts: The case of gold-palladium nanoalloys, Cataly. Sci. Technol., 7 (2017) 1848-1856. 
Figure captions

Fig. 1 Morphology and structure characterizations of Au@Pd BNFs. SEM image (a), EDX mappings (b), TEM image and XRD patterns (d) of Au@Pd BNFs.

Fig. $2 \mathrm{UV}$-vis spectra for the $\mathrm{W}$ from the synthesis terminated at different reaction times $(t=0,1,4,8,12,24 \mathrm{~h})$. All solutions were diluted for 22 times. Insets: enlarged figures for the red regions.

Fig. 3 Schematic of the interfacial reaction mechanism for Au@Pd BNFs.

Fig. 4 (a) CVs of Au@Pd BNFs and Pd/C in a $1 \mathrm{M} \mathrm{NaOH}$ solution at $50 \mathrm{mV} / \mathrm{s}$ with the current normalized by the loading amount of Pd (3.5 $\mu$ g for Pd NFAs and $1.0 \mu \mathrm{g}$ for Pd/C); (b) CVs of Au@Pd BNFs and Pd/C in a $1 \mathrm{M} \mathrm{NaOH}+1 \mathrm{M}$ ethanol solution at $50 \mathrm{mV} / \mathrm{s}$ with the current normalized by the EASA of Pd; (c) CAs of Au@Pd BNFs and $\mathrm{Pd} / \mathrm{C}$ in a $1 \mathrm{M} \mathrm{NaOH}+1 \mathrm{M}$ ethanol solution at an applied voltage of $-0.3 \mathrm{~V}$ for $2000 \mathrm{~s}$ with the current normalized by the EASA of Pd. The catalytic performance of Au@Pd BNFs and Pd/C is compared with the first scan.

Fig. 5 CVs of Au@Pd BNFs in a $1 \mathrm{M} \mathrm{NaOH}$ solution (a) and in a $1 \mathrm{M} \mathrm{NaOH}+1 \mathrm{M}$ ethanol solution (b) for the $1^{\text {st }}$ (dashed) and $100^{\text {th }}$ (solid) cycles. (c) The plot of $I_{\mathrm{f}}$ in Fig. S16(b) against cycle number. The currents are normalized with the loading amount of of Pd. Scan rate: $50 \mathrm{mV} / \mathrm{s}$. TEM images of Au-Pd BNFs before (d) and after (e) the EOR cycling for 100 times. The corresponding schematics are shown in the right bottom corner. 